\title{
Effect of Composite Edible Coating on Antimicrobial and Antioxidant Properties of Sapota
}

\author{
Adrita Banerjee* and Jincy Abraham \\ Department of Food Science and Nutrition Management, J.D. Birla Institute (Affili- \\ ated to Jadavpur University), Kolkata, India \\ *Corresponding Author: Adrita Banerjee, Department of Food Science and Nutrition \\ Management, J.D. Birla Institute (Affiliated to Jadavpur University), Kolkata, India.
}

Received: July 23, 2021

Published: August 12, 2021

(C) All rights are reserved by Adrita Banerjee and Jincy Abraham.

\begin{abstract}
Composite edible coatings made up of natural gums and polysaccharides contribute to extending the shelf life and reducing spoilage of fresh produces. This research work was designed with the objective to determine the effect of a composite edible coating on sapota fruit. The coating was effective in minimizing weight loss and overall spoilage of the fruit. Physical and microbial assay of whole sapota fruits coated with of the developed coating was performed. The objective of this work was also to study the consumer awareness and acceptance of edible coatings on fruits. The results of the survey showed that consumers majorly preferred edible coatings due to enhanced stability and better quality. Socio-economic status, age, knowledge level of the consumers influenced their preference and acceptability of edible coatings. This study demonstrates the use of composite multilayered edible coatings as a potential means to decrease the post harvest losses for sapota fruit and increase its shelf life.
\end{abstract}

Keywords: Basil Seed Gum; Composite Edible Coating; Sapota; Post Harvest Loss; Consumer Acceptance

\section{Introduction}

Harmful effects of synthetic packaging on human health and environment have made way for the emergence of biodegradable edible packaging. Edible packaging broadly denotes edible films and coatings. Edible films and coatings may be defined as protective layers created around food surface by applying solutions made from edible polymers like polysaccharides, proteins, lipids or their combinations. The protective layer thus created, acts as a barrier between the food surface and spoilage causing factors thereby enhancing the shelf life of the food. This results in improvement of gas and moisture barrier properties, mechanical properties, sensory quality and even the nutritional characteristics of coated or wrapped food. An edible coating or film is formulated with substances that are legal as per the standard food laws and must not interfere with the sensory profile or other quality attributes of the food. It may also be used as a carrier for bioactive compounds like flavors, nutraceuticals, antimicrobials etc. which improves the functional or nutritional qualities of the coated food.
Recent advances related to edible films and coatings have resulted in significant improvement of their physical and barrier properties thus paving the way for their consideration as an alternative to synthetic packaging materials.

The disadvantages of the techniques being used to preserve fresh produce and increasing negative environmental impact have created urgency for the invention of alternative packaging techniques such as edible coatings. Many years of research have been conducted to develop a material that would coat fruit so that an internal modified atmosphere would develop. Studies have shown that ripening can be retarded, color changes can be delayed, water loss and decay can be reduced, and appearance can be improved by using a simple and eco friendly technology, edible coating.

Edible coatings produced from polysaccharides (cellulose, starch, pectins, seaweeds, gums, chitosan and pullulan) have been widely used in the food industry in the last few years, while lig- 
nocellulosic materials have been recently proved to be suitable for edible coating production. On food models, it was found that edible coatings based on bicomponent formulations demonstrate effective microbial spoilage inhibition. Composite coatings did not obstruct fruit gas exchange or caused accumulation of off flavor volatiles [1]. Polysaccharide gums have been recently studied in their possibilities as edible coatings and films promoters due to their sustainable, biodegradable and bio-compatible characteristics. The seeds of Ocimum basilicum L., also known as basil, contain a considerable amount of gum composed by two major fractions of polysaccharides ( $43 \%$ glucomannan and $24.3 \%$ xylan) with outstanding functional properties in developed edible films. Studies on physico-mechanical properties of basil seeds reported optimal extraction conditions of gum from basil seeds, in terms of $\mathrm{pH}$, temperature and water/seed ratio [2].

In India, fruits are grown on an area of 5.78 million hectares with a production of 63.50 million tonnes. The important fruits are mango, citrus, banana, sapota, apple, litchi, guava and pomegranate. Despite a phenomenal increase in the production of fruits and vegetables, the much needed nutritional status of the population has not improved much. One of the main reasons for low per capita availability is the enormous losses of fruits which are estimated to be 15 to $50 \%$ that occur at different stages of handling, transport, storage, processing and distribution [3]. Sapota is a climacteric fruit characterized by its rapid postharvest ripening and senescence followed by spoilage is due to high rate of ethylene liberation. So far, there has been no cited literature on the effect of composite edible coatings on sapota fruit with usage of basil seed gum as a novel ingredient, hence in this regard, a research work was undertaken to develop a composite edible coating using basil seed gum extract as a novel ingredient, along with xanthan and tragacanth gum and to study its effect on the physio-chemical, antimicrobial and antioxidant properties of sapota.

Research has previously been conducted to establish the benefits of edible coatings in increasing market opportunity for fruits, as well as in extending their post harvest shelf life. But little research has so far examined the consumer acceptance of these products, especially regarding their application on widely used fruits. Therefore, a Knowledge, Attitude and Practice (KAP) survey was also conducted to understand the consumer acceptance of edible coatings.

\section{Materials and Methods}

\section{Materials}

The basil seeds, xanthan gum and tragacanth gum were obtained from the local market in Kolkata, West Bengal, India. Sapota (Manilkara achras Mill.cv. Kalipatti) fruits were obtained from a commercial fruit grower at Baruipur, West Bengal.

\section{Extraction of basil seed gum}

This procedure was followed to extract gum from basil seeds. Swollen seeds were stirred in a hot plate magnetic stirrer. Separation of the gum from the swollen seeds was achieved by cold centrifugation followed by manual filtration with cheesecloth to filter out any insoluble residue present.

\section{Preparation of the coating}

To prepare the coating solution, $0.1 \mathrm{M} \mathrm{NaOH}$ solution was added drop wise to all the basil seed gum extracts until a $\mathrm{pH}$ of 9 was reached. Similarly, a solution was made of commercial xanthan gum powder with double sterilized water (water to xanthan gum ratio-10:1). Glycerol ( $6 \% \mathrm{w} / \mathrm{w})$ was added as a plasticizer in all the solutions. The solutions were then stirred on a magnetic stirrer for 30 minutes to obtain uniform solution.

\section{Preparation of the sapota fruits for coating application}

The fruits were first washed in a solution containing sterilized water and sodium hypochlorite as a sanitiser. They were then divided into three sets $(5,10$ and 15 layers) of coating treatments each having three variations with one control as follows:

- Variation 1: Xanthan gum (XG)

- Variation 2: Xanthan gum + basil seed gum extract $(\mathrm{XG}+\mathrm{BG})$

- Variation 3: Xanthan gum + basil gum extract + tragacanth gum $(\mathrm{XG}+\mathrm{BG}+\mathrm{TG})$

- Control: Sterilized water + sanitizer (without any coating).

\section{Application of the coating on sapota}

Each batch of sapota fruits were subjected to coating treatments by the method of dipping. Post dipping, samples were stored in sterilized zip lock bags at room temperature and under dry conditions for further analysis. 
Physico-chemical and microbial analysis of the sapota samples

The coated and control sample were analysed to measure parameters such as weight loss percentage (WLP), colour, $\mathrm{pH}$, total soluble solids (TSS), total phenol content and antioxidant capacity to interpret the effectiveness of the developed composite coating on the sapota samples. Microbiological analysis of the coated and control fruits was carried out using the method described by The International Commission on Microbiological Specifications for Foods (ICMSF).

\section{Shelf life}

The shelf life of fruits was calculated by counting the days required for the fruit to reach the last stage of ripening, but up to the stage of their marketability. The shelf life of the fruits was considered on the basis of their visual appearance.

Statistical analysis of the results obtained

Statistical analysis was performed using SPSS Statistics (Version 26). Analysis of Variance (ANOVA) was used to assess the statistical significance at $\mathrm{P}$-value $<0.05$.

\section{KAP survey procedure}

A Knowledge, Attitude and Practice Questionnaire was prepared with ten relevant questions in each section. The questions were framed on the basis of estimating consumer knowledge and attitude towards edible coatings.

\section{Results and Discussions}

The coated and uncoated sapota samples were analysed for different quality parameters.

\section{Weight loss}

There was a significant weight loss for the control sample at the end of the storage period, and the weight loss percentage was higher compared to the coating treatments. There was no significant weight loss in the coated samples until the $8^{\text {th }}$ day of storage while the uncoated samples (control) lost weight much faster.

The results obtained indicated that the variation having combination of three gums, Xanthan, Basil seed and Tragacanth significantly $(\mathrm{p}<0.05)$ reduced weight loss and acted as a barrier against water loss from sapota.

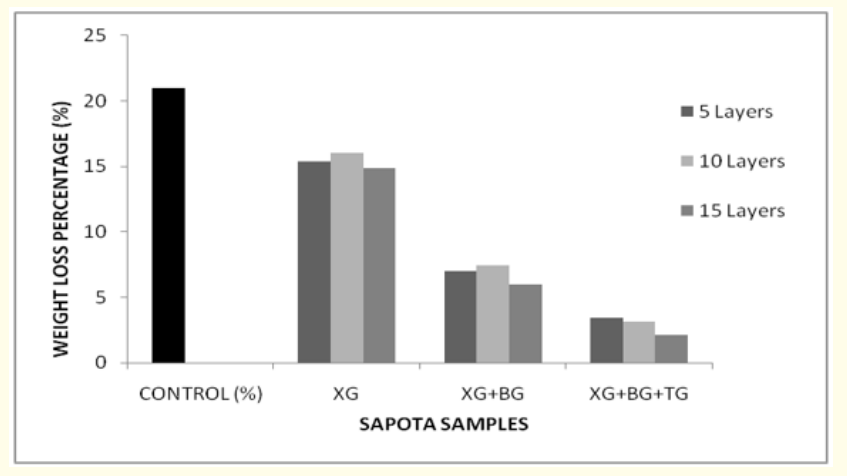

Figure 1: Graph represents the Weight loss \% between 5, 10 and 15 layer coating treatments.

\section{Colour measurements}

All coating treatments significantly retained the color of the fruit as compared to the uncoated control sample. The reduction in lightness $\left(\mathrm{L}^{*}\right)$ values were low in the coated sapotas during storage time whereas it was higher in the control sample starting from the third day of storage. This could refer to the changes in epicuticular wax of the fruit as a result of the varying coating treatments. Ripening of sapota fruit is associated with loss of carotenoids and chlorophyll. The relatively higher $\mathrm{L}^{*}$ value of the coated sapotas in this study could be attributed to the ability of edible coatings in delaying chlorophyll catabolism and synthesis of carotenoids.

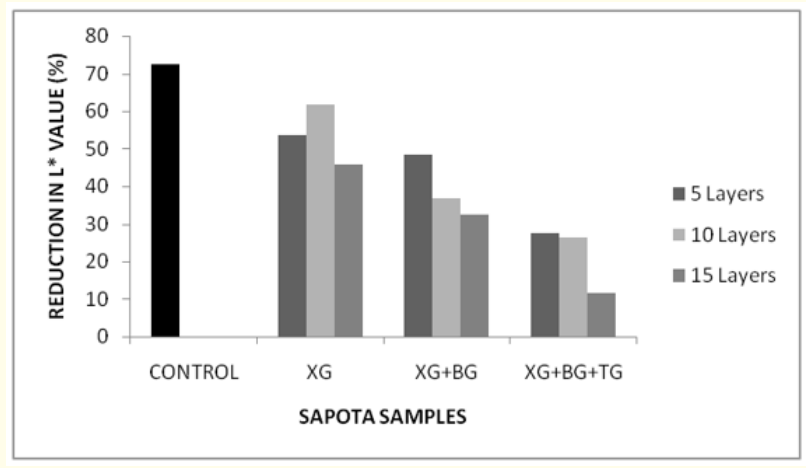

Figure 2: Changes in colour (lightness, $\mathrm{L}^{*}$ ) of coated and uncoated Sapota samples (XG- Xanthan Gum, XG+BG- Xanthan and Basil Seed Gum, XG+BG+TG - Xanthan, Basil Seed and Tragacanth Gum). 
The results clearly describe the effectiveness of the composite coating in improving the aesthetic look of the sapota fruit by maintaining the higher $\mathrm{L}^{*}$ values till the end of the storage period. Similar findings were reported where tomatoes coated with gum arabic delayed color change due to reduced respiration rates.

\section{pH Measurement}

Coated sapota fruit retained higher $\mathrm{pH}$ values, as compared to the control. The changes in $\mathrm{pH}$ values in all three coating treatments showed variation during their storage period. It was consistently decreasing in the control sample with the increase of storage time and reached to the minimum value at the end of storage period.

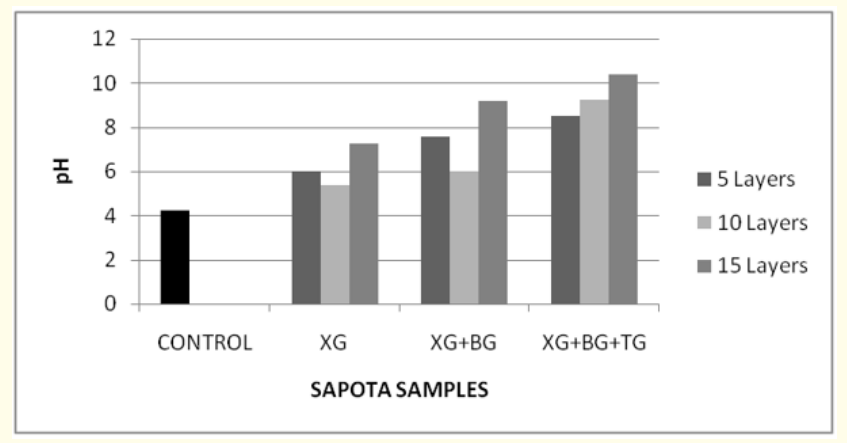

Figure 3: Changes in $\mathrm{pH}$ of coated and uncoated samples between three coating treatments (XG- Xanthan Gum, XG+BG-Xanthan and Basil Seed Gum, XG+BG+TG - Xanthan, Basil Seed and Tragacanth Gum).

The 15 layer coating treatment was found to be most effective in preventing the decrease of acidity thereby maintaining the $\mathrm{pH}$ levels.

Total soluble solids (TSS)

Total Soluble Solids content of coated sapota samples and control (uncoated) sample during their storage increased significantly in the control, but was low in case of the 15 layer coating treatments at the end of storage time.

The lower total soluble solids values of the coated samples at the end of storage period as compared to the control may be attributable to the fact that the immersion of the sapotas in $\mathrm{XG}+\mathrm{BG}+\mathrm{TG}$ coating may have retarded the rate of respiration, thereby sup-

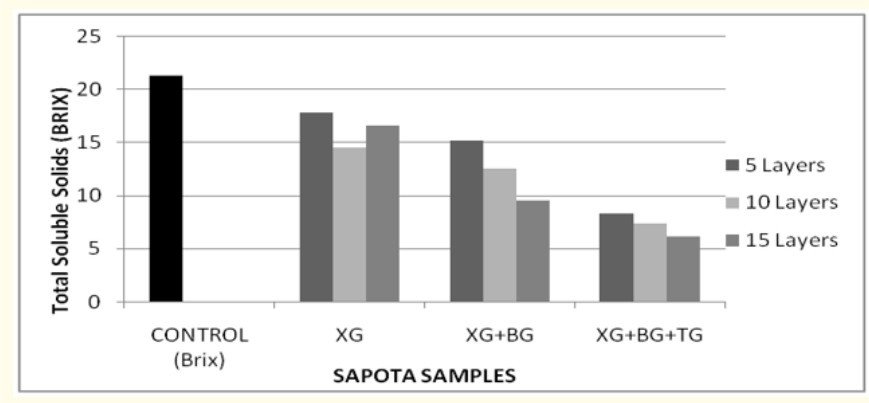

Figure 4: Changes in Total Soluble Solids of coated and uncoated samples (XG-Xanthan Gum, XG+BG- Xanthan and Basil Seed Gum, XG+BG+TG - Xanthan, Basil Seed and Tragacanth Gum).

pressing the synthesis and use of metabolites, resulting in lower soluble solids due to slower hydrolysis of carbohydrates to sugars.

Total phenol content (TPC)

Changes in total phenolic content in the coated and control samples were determined during storage period (Graph 5).

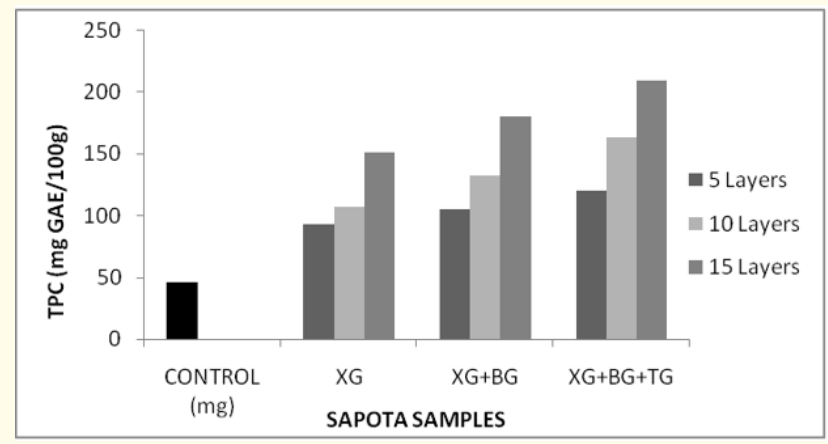

Figure 5: Changes in Total Phenol Content in coated and uncoated samples (XG-Xanthan Gum, XG+BG- Xanthan and Basil Seed Gum, XG+BG+TG - Xanthan, Basil Seed and Tragacanth Gum).

After two days of storage, the content of total phenolics was estimated higher in the coated samples than that measured in the control. This trend continued till the end of storage period, where the phenol content reduced maximum in the control, whereas it was significantly $(\mathrm{p}<0.05)$ increased in the 15 layer coated samples. 


\section{Antioxidant capacity}

It was observed that the coating treatments had a significant $(\mathrm{p}$ $<0.05$ ) effect on the antioxidant capacities of the sapota samples, whereas the control sample did not show a desirable antioxidant level.

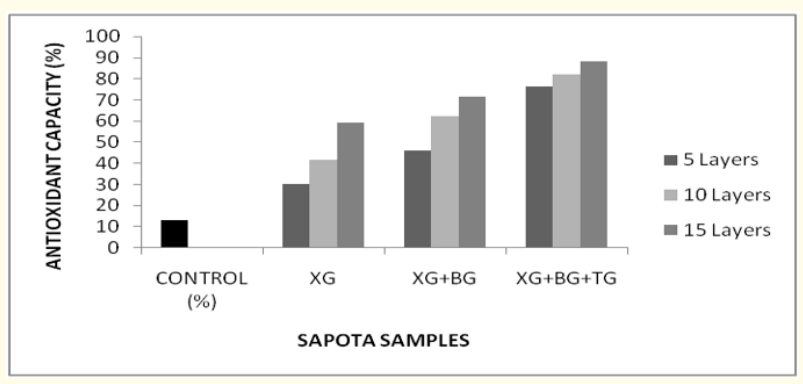

Figure 6: Changes in antioxidant activity in coated and uncoated Sapota samples.

The edible coatings of $\mathrm{XG}+\mathrm{BG}+\mathrm{TG}$ prevented the loss of antioxidant capacity of the fruit, and also maintained it throughout the storage period which could be attributable to the ability of this composite coating to enhance the antioxidant capacity.

\section{Microbial analysis}

The composite edible coating exhibited potential to be an effective antimicrobial agent.

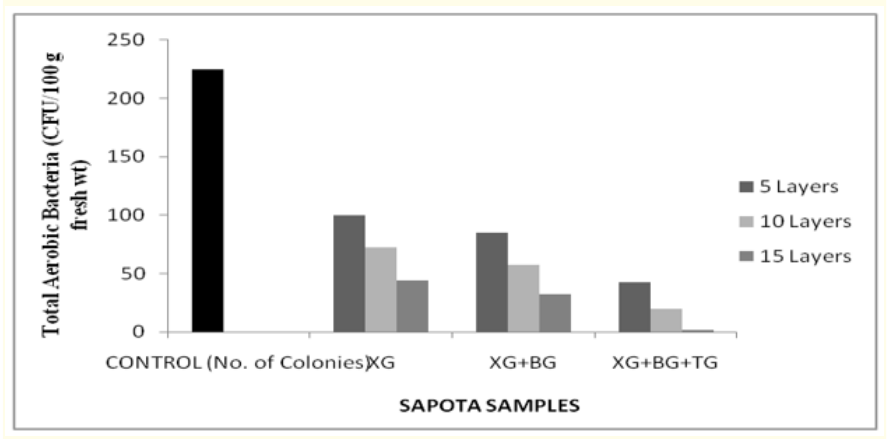

Figure 7: Changes in activity of Total Aerobic Bacteria in coated and uncoated sapota samples.

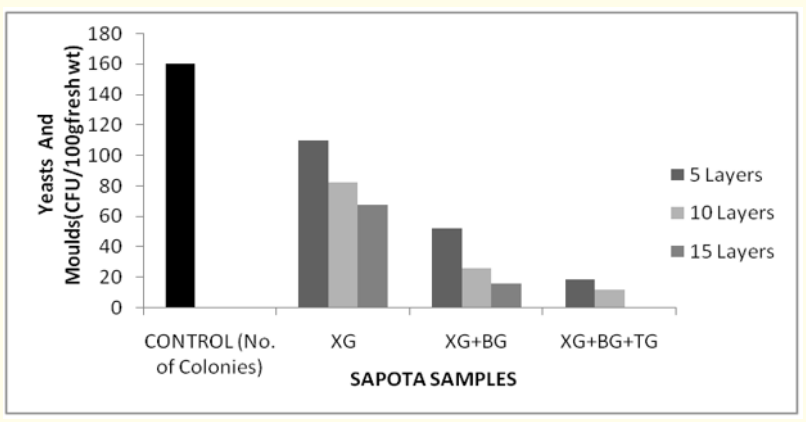

Figure 8: Changes in activity Yeasts and Moulds in coted and uncoated sapota samples at the end of storage period (XG-Xanthan Gum, XG+BG- Xanthan and Basil Seed Gum, XG+BG+TG - Xanthan, Basil Seed and Tragacanth Gum).

The 15 layer coating treatment showed significant $(\mathrm{p}<0.05)$ efficiency in preventing microbial spoilage at the end of eight day storage period in both the agar mediums. This lower count of both the types of microorganisms in the coated samples indicate decreased microbial growth throughout the storage period at room temperature thereby enhancing the shelf life of the sapotas up to ten days.

\section{Shelf life analysis}

The combined effect of xanthan, tragacanth and basil seed gum altogether with the 15 coats showed the most effective result by delaying the ripening and extending the shelf life up to ten days at room temperature as compared to the control sample which was spoiled after the third day of storage.

The second part of this study includes findings of the KAP survey conducted to understand the consumer acceptability of edible coatings.

Consumer perception of edible coatings based on their knowledge, attitude and practice levels

The survey was based on estimating the overall knowledge, attitude and practice levels of the consumers towards accepting the application of edible coatings on fresh produce especially fruits. The results revealed that the overall knowledge levels of the consumers about edible coatings as a packaging and preservation method depends on different factors such as their socio economic status, 
education, age and awareness levels. Consumers from high income group were more inclined to using edible coatings on a widespread level. It was observed that most of the consumers above the age of 25 did not want to buy the highly priced edible coatings or were not aware of its applications.

Almost $65 \%$ of the respondents were aware of the use of edible coatings and films as a packaging method. This could be due to the increased level of awareness among people regarding advancements in the field of sustainable food packaging and preservation techniques publicized through mass media. Some of the respondents indicated that they have heard about edible coatings but they were not sure of the composition of the coatings and their exact application. Although, rest of the respondents did not know about edible coatings in general, indicating a lack of knowledge.

Results showed that many of the consumers believed that edible coating could be an effective means of decreasing post harvest losses of fruits by acting as a natural packaging material. They know the use of edible coating as an alternative means of packaging fresh fruits which indicated good knowledge levels among them. About $60 \%$ of the consumers had knowledge of the added benefits of using edible coatings in fruits. They were aware of the presence of various bioactive and functional components in the natural coating applications. The respondents, who answered negatively, knew that edible wax was used to coat some fruits like apples, but were not sure of the specific benefits of the wax. When asked if they were willing to buy the coated fruits at a higher price than normally available in the market, about $70 \%$ responded positively. This suggests that these consumers are aware of the added benefits of the edible coatings that naturally increase its cost when compared to normal fresh fruits available in the market.

Overall, it was found from the knowledge, attitude and practice survey that currently consumers who are conscious about their eating habits and are aware of the recent advancements in the field of health and technology, are willing to accept the widespread use of edible coatings on fresh produce and also believe that it is going to benefit them. Those who were not aware of the presence of edible coatings were either skeptical of accepting this new technique or already had a pre-conceived notion about it. They perceived edible coatings to be artificial in nature and some even viewed it as adulteration [4-16].

\section{Conclusion}

Improper post harvest treatment lead to a shorter shelf-life of fresh produces like fruits and vegetables leading to food wastage. Edible coatings come as an environment friendly solution to the problem of food spoilage. This study was thus designed to investigate the possible effects of a composite edible coating on the antimicrobial and antioxidant properties of Sapota fruit and to understand the consumer acceptance of edible coatings on fruits. On the basis of the results obtained it is concluded that the composite edible coating is effective in enhancing the shelf life and retaining the physio-chemical quality parameters of sapota. It is also seen that the coating is most effective when it is applied in multi layers, which is in this study, the 15 layer treatment that turned out to have the most potential in controlling microbial spoilage and maintaining the shelf life. It can be concluded from the Knowledge, Attitude and Practice survey conducted on consumer acceptance of edible coatings that the industry should put emphasis on marketability and commercialization of this natural and alternative source of post harvest preservation and packaging, since there is sufficient knowledge amongst consumers, but a lack of awareness of the usage.

\section{Bibliography}

1. Poverenov E and Arnon-Rips H. "Improving food products' quality and storability by using Layer by Layer edible coatings". Trends in Food Science and Technology 75 (2018): 81-92.

2. Razavi S M A., et al. "Image processing and physico-mechanical properties of basil seed (Ocimum basilicum L.)". Journal of Food Process Engineering 33.1 (2010): 51-64.

3. Gajanana TM., et al. "Post Harvest handling, marketing and assessment of losses in papaya". Acta Horticulturae 851 (2010): 519-526.

4. Ali A., et al. "Effect of gum arabic as an edible coating on antioxidant capacity of tomato (Solanum lycopersicum L.) fruit during storage". Postharvest Biology and Technology 76 (2013): 119-124.

5. Amiot MJ., et al. "Influence of cultivar, maturity stage, and storage conditions on phenolic composition and enzymatic browning of pear fruits". Journal of Agricultural and Food Chemistry 43 (1995): 1132-1137. 
6. Azoma J and Sakamoto M. "Cellulosic hydrocolloid system present in seed of plants". Trends in Glycoscience and Glycotechnology 15 (2003): 1-14.

7. Ball J A. "Evaluation of two lipid-based edible coatings for their ability to preserve post harvest quality of green bell peppers". Blacksburg, Virginia, USA: Faculty of the Virginia Polytechnic Institute and State University. Master Thesis (1997).

8. Bolin H R and Huxsoll CC. "Control of minimally processed carrot (Dascuscarota) surface discoloration caused by abrasion peeling". Journal of Food Science 56 (1991): 416-418.

9. Carneiro-da-Cunha MG., et al. "Physical properties of edible coatings and films made with a polysaccharide from Anacardium occidentale L". Journal of Food Engineering 95 (2009): 379-385.

10. Cui S W M., et al. "Seed polysaccharide gums". In C. G.Biliaderis, \& M. S. Izydorczyk (Eds.). Functional food carbohydrates (2007): 127-158.

11. Debeaufort F., et al. "Edible films and coatings:tomorrow's packagings: A review". Critical Reviews in Food Science and $\mathrm{Nu}$ trition 38.4 (1998): 299-313.

12. Mathews S., et al. "Ocimum basilicum: A new non-conventional source of fibre”. Food Chemistry 47 (1993): 399-401.

13. Mohamed AYI., et al. "Utilization of edible coating in extending the shelf life of minimally processed prickly pear". Journal of Applied Sciences Research 9 (2013): 1202-1208.

14. Rojas-Grau M A., et al. "Edible coatings to incorporate active ingredients to fresh-cut fruits: a review". Trends in Food Science and Technology 20 (2009): 438-447.

15. Stathopoulos CE., et al. "Effect of edible coatings on some quality characteristics of sweet cherries". International Food Research Journal 18 (2011): 1237-1241.

16. Xu Q., et al. "Effect of chitosan coating and oil fumigation on the microbiological and quality safety of fresh-cut pear". Journal of Food Safety 33 (2013): 179-180.

\section{Volume 5 Issue 9 September2021}

(C) All rights are reserved by Adrita Banerjee and Jincy Abraham. 